\title{
DISERTACIONES
}

Grupos minoritarios y estigmatizados: diversidad funcional, religiosa, étnica, afectivo-sexual o de identidad de género en la comunicación

ISSN: $1856-9536$

Doi: https://doi.org/10.12804/revistas.urosario.edu.co/disertaciones/a.11148

Volumen 15, Número 1 / Enero-junio 2022

Versión PDF para imprimir desde

http://revistas.urosario.edu.co/index.php/disertaciones

Para citar este artículo: Martín, T., \& Chaves Vázquez, B. (2022). La influencia de Instagram en la crea-

\section{LA INFLUENCIA DE INSTAGRAM EN LA CREACIÓN Y REPRODUCCIÓN DEL IDEAL DE BELLEZA FEMENINO}

\author{
The Impact of Instagram on the Creation and Reproduction of Female \\ Beauty Ideals
}

A influência do Instagram na criação e reprodução do ideal de beleza feminina

Teresa Martín, Universidad de Salamanca (España)

teresam@usal.es

Beatriz Chaves Vázquez, Universidad de Salamanca (España)

beatrizchaves@usal.es

Recibido: 27 de septiembre del 2021

Aprobado: 1 de noviembre del 2021

Fecha de prepublicación: 14 de diciembre del 2021

\section{RESUMEN}

Las redes sociales hacen gala de una gran presencia en la sociedad contemporánea, afectando incluso cómo percibimos los cánones de belleza. Este trabajo estudia la posible influencia del fenómeno de los influencer en la reproducción del ideal de belleza femenino, analizando los contenidos publicados en Instagram por algunas de estas figuras públicas e indagando en el tipo de uso que hacen las personas anónimas de esta red social. Para ello, se ha recurrido a una metodología mixta compuesta por un análisis de contenido cualitativo de las publicaciones y una encuesta en línea ( $n=509)$. La presente investigación ha permitido comprobar, entre otros aspectos, cuál es el patrón de belleza que impera en Instagram y las posibles evoluciones sobre el canon de belleza "tradicional", 


\title{
DISERTACIONES
}

Grupos minoritarios y estigmatizados: diversidad funcional, religiosa, étnica, afectivo-sexual o de identidad de género en la comunicación

ISSN: 1856-9536

Doi: https://doi.org/10.12804/revistas.urosario.edu.co/disertaciones/a.11148

Volumen 15, Número 1 / Enero-junio 2022

Versión PDF para imprimir desde

http://revistas.urosario.edu.co/index.php/disertaciones

dictaminando si existen implicaciones de género en dichos estereotipos. El trabajo advierte sobre el impacto de los influencers en la creación o mantenimiento de estereotipos que afectan a la autoimagen.

Palabras clave: Instagram; redes sociales; influencers; estereotipos de género; cánones de belleza.

\begin{abstract}
Social media has such a strong presence in contemporary society, even affecting how we perceive beauty standards. For this reason, this paper analyses significant phenomena such as influencers, by studying the content published by some of these public figures. To this end, an online survey $(n=509$ people) and a qualitative content analysis were carried out as tools for observing the beauty standards represented in the publications shared by users on social media. This research has made it possible to verify, among other elements, what is the prevailing pattern of beauty on Instagram, as well as possible evolutions of the "traditional" beauty standard, determining whether there are gender implications in these stereotypes. The work warns about the impact of influencers in the creation or maintenance of stereotypes that affect self-image.
\end{abstract}

Keywords: Instagram; social media; influencers; gender stereotypes; beauty standards.

\section{RESUMO}

As redes sociais têm grande presença na sociedade contemporânea, afetando inclusive a forma como percebemos os padrões de beleza. Este trabalho estuda a possível influência do fenômeno dos influenciadores na reprodução do ideal de beleza feminina, analisando o conteúdo publicado no Instagram por algumas dessas figuras públicas e investigando o tipo de uso que os anônimos fazem dessa rede social. Para tanto, foi utilizada uma metodologia mista, que consiste em uma análise qualitativa de conteúdo das publicações e uma pesquisa online $(n=509)$. Este trabalho permitiu-nos verificar, entre outros aspectos, qual é o padrão de beleza que prevalece no Instagram, as possíveis evoluções no cânone da beleza «tradicional», determinando se existem implicações de género nos referidos estereótipos. 0 trabalho adverte sobre o impacto dos influencers na criação ou manutenção de estereótipos que afetam a autoimagem.

Palavras-chave: Instagram; redes sociais; influenciadores; estereótipos de gênero; cânones de beleza. 


\section{DISERTACIONES}

ESTUDIOS

Grupos minoritarios y estigmatizados: diversidad funcional, religiosa, étnica, afectivo-sexual o de identidad de

género en la comunicación

ISSN: $1856-9536$

Doi: https://doi.org/10.12804/revistas.urosario.edu.co/disertaciones/a.11148

Volumen 15, Número 1 / Enero-junio 2022

Versión PDF para imprimir desde

http://revistas.urosario.edu.co/index.php/disertaciones

\section{Introducción}

Los medios de comunicación y su vinculación con el ámbito de la publicidad han tenido un importante peso en la creación y perpetuación de estereotipos de género en nuestra sociedad. El auge de las redes sociales ha motivado el surgimiento de figuras como los influencers. La influencia social que ejercen este tipo de canales digitales ha propiciado un considerable aumento de los estudios focalizados en estos canales digitales (Rodríguez et al., 2017). La expansión de las redes sociales actúa como un mecanismo de transmisión (Garlick, 2020), contribuyendo a la generación de estereotipos de esta índole, posibilitando que surjan fenómenos como los influencers o que se popularice el uso - principalmente en Instagram - de herramientas como los filtros de belleza, que editan los rostros de quienes los usan (Tolentino, 2019). Dentro de este ámbito, uno de los aspectos que suscita más interés es el estudio de la representación de la belleza por sus posibles implicaciones en la conformación y mantenimiento de ciertos estereotipos de género vinculados al ámbito femenino (Jiang \& Ngien, 2020; Keyte et al., 2020; Lup et al., 2015; O'Brien, 2015; Reece \& Danforth, 2017). En este sentido, muchos estudios sugieren que este tipo de uso de las redes sociales entrañan consecuencias y daños sobre la percepción de la propia imagen, en la autoestima, en el estado de ánimo o en la insatisfacción corporal (McLean et al., 2015). Además, diversas investigaciones apuntan que determinados usos de las redes sociales pueden generar otras conductas como dependencia a la validación externa, a través de la obtención de likes y comentarios de otras personas (Lowe-Calverley et al., 2019; Tiggemann et al., 2018; Jiang \& Ngien, 2020; Tiggemann \& Barbato, 2018).

Las tendencias a compararse con otras personas y a objetivar sexualmente el propio físico pueden explicarse si recurrimos a las teorías de la comparación social -formulada por Festinger en 1954-y de la auto objetivación (Fredrickson \& Roberts, 1997). La primera de estas teorías afirma que las personas se evalúan a sí mismas estableciendo comparaciones entre sus propias habilidades y destrezas con las de los demás (Festinger, 1954). Cuando esta clase de comparación conduce a una autoimagen negativa, los sujetos envidian a quienes poseen aquello que ellos anhelan (Smith \& Kim, 2007). Así mismo, Lim y Yang (2015) demostraron que existe una asociación estadísticamente significativa entre la comparación social y la envidia: cuando más nos comparamos con otros individuos, más los envidiamos.

La teoría de la auto objetivación plantea que las mujeres y las niñas suelen estar acostumbradas a interiorizar las perspectivas de terceros como óptica primaria con la cual observar sus físicos (Fredrickson \& Roberts, 1997); es decir, se produce una separación entre el individuo y su cuerpo -en otras palabras, la objetivación posibilita que se evalúe el cuerpo de una mujer, sus partes o sus funciones aislándolas de la propia mujer y se actúe como si realmente representaran todo lo que es dicha mujer, ignorando sus cualidades no físicas, sus ideas o sus habilidades; la mujer acaba internalizando esto y empieza a considerarse como un objeto que ha de ser observado y evaluado- (Feltman \& Szymanski, 2018).

Por todo ello, se hace especialmente necesario acercarse a este estudio desde una perspectiva de género, siguiendo la línea de trabajos anteriores desarrollados en España (Martín García, 2017; Riera \& Figueras, 2012; Ruíz, 2012; Menéndez \& Figueras, 2014; Fernández, 2013). Al mismo tiempo, la evolución de las redes sociales está generando mucha preocupación social e institucional a nivel global. Es el caso del Reino Unido en donde el organismo que regula la publicidad en dicho país -Advertising Standards Authority (ASA) - obliga a las marcas e 


\section{DISERTACIONES}

ESTUDIOS

influencers (desde febrero del 2021) a prescindir de los filtros (García, 2021). En el 2017, Francia aprobó una ley que exige que cualquier foto que sea usada con fines comerciales lleve un distintivo que indique que ha sido retocada o editada, para poder luchar contra los trastornos de la conducta alimentaria y los estándares difíciles de alcanzar (Daldorph, 2017). Actualmente en España no existe ningún tipo de regulación semejante en cuanto a retoques, la responsabilidad cae sobre las agencias y marcas. Sin embargo, en enero del 2021 entró en vigor el Nuevo código de conducta creado por la Asociación Española de Anunciantes (AEA) y la Asociación para la Autorregulación de la Comunicación Comercial (Autocontrol), con el fin de que las publicaciones en redes sociales hechas por influencers -u otras figuras públicas - incluyan un distintivo o identificación que detalle que se ha realizado con fines publicitarios (Ortiz, 2021).

Por todo ello, la presente investigación pretende adentrarse en el patrón de belleza que impera en Instagram, así como analizar si se ha producido alguna evolución sobre el canon de belleza "tradicional", dictaminando si existen implicaciones de género en dichos estereotipos.

\section{Metodología}

El principal objeto de investigación de este trabajo es el análisis y estudio de los cánones de belleza representados en las publicaciones compartidas por los usuarios de Instagram. Se pretende comprobar el patrón de belleza que impera en dicha red social, determinar si se reproducen estereotipos asociados al ámbito femenino, y en qué medida estos aspectos pueden tener un impacto en las personas que siguen este tipo de cuentas en redes sociales. Para llevar a cabo este estudio, se tomaron como punto de partida las siguientes hipótesis:

- $\mathrm{H}_{1}$ : las cuentas de influencers/instagrammers más populares perpetúan el ideal de belleza tradicional (juventud, rasgos caucásicos, entre otros elementos) y pueden influir en la imagen física que proyectan sus seguidores.

- $\mathrm{H}_{2}$ : el ámbito influencer está ligado al consumo de productos de moda y belleza.

- $\mathrm{H}_{3}$ : los influencers de este ámbito crean publicaciones en Instagram que refuerzan ciertos estereotipos femeninos (hipersexualización, interés en moda y belleza), que influyen en los patrones de conducta de usuarios anónimos de esta red social.

La metodología empleada para desarrollar esta investigación está basada en los diseños mixtos (Johnson \& Christensen, 2008; Onwuegbuzie et al., 2009; Teddlie \& Tashakkori, 2009; Johnson \& Onwuegbuzie, 2004; Onwuegbuzie \& Johnson, 2006, todos ellos, citados en Baltar et al., 2011), empleando tanto métodos cuantitativos como cualitativos.

El análisis se ha planteado en dos fases que se complementan mutuamente: la primera comprende la realización de un análisis de contenido cualitativo (Krippendorff, 1980, p. 28, citado en Porta \& Silva, 2003), que permite estudiar los contenidos publicados en la red social Instagram por influencers. La segunda consiste en distribuir una encuesta disponible en línea en la plataforma Qualtrics con la que se pretende conocer qué tipo de uso hacen las personas anónimas (las personas que no son influencers) de esta red social y comprobar en qué medida puede estar condicionado por los contenidos generados por influencers o figuras públicas. 


\section{DISERTACIONES}

Grupos minoritarios y estigmatizados: diversidad funcional, religiosa, étnica, afectivo-sexual o de identidad de género en la comunicación

\section{Muestra}

La muestra del análisis de contenido cualitativo se compone de un total de 240 publicaciones de Instagram, pertenecientes a un conjunto de cinco personajes públicos (influencers). Para determinar su elección, se partió de un estudio realizado por la Fundación Marqués de Oliva en colaboración con Mercad2 sobre los influencers con más seguidores en España "El Estudio de los 500 españoles más influyentes", que se compone de catorce categorías y engloba a un total de 500 personas (Fundación Marqués de Oliva, 2021). Dentro de esos ámbitos está el relativo a los influencers, el cual cuenta con un total de 50 personas. En dicha categoría se han extraído las cinco cuentas con más seguidores para incluirse en la muestra, a saber:

Tabla 1. Muestra

\begin{tabular}{ccc} 
& Número de seguidores & Temática(s) \\
Alexandra Pereira/Lovelypepa (@alexandrapereira) & 2 millones & Viajes, fitness, moda \\
\hline Laura Escanes (@lauraescanes) & 1.5 millones & Moda, belleza \\
\hline Patry Jordan (@patryjordan) & 1.4 millones & Moda, belleza, fitness \\
\hline Sara Escudero (@collagevintage) & 1.1 millones & Moda, viajes \\
\hline Rocío Camacho (@rocioccamacho) & $659 \mathrm{k}$ & Moda, belleza \\
\hline
\end{tabular}

Fuente: elaboración propia.

Se han estudiado un total de cuatro publicaciones mensuales de cada una de estas influencers durante un periodo de doce meses comprendido entre abril del 2020 y marzo del 2021, excepto en el caso de Patry Jordán, en el cual no se pudo establecer este criterio de cuatro publicaciones al mes debido a la falta de regularidad en sus patrones de subida de contenidos a Instagram, y por tanto, en algunos meses se trabajó con más material que en otros, con el objetivo de alcanzar la cuota individual de cuarenta y ocho. Es necesario especificar que el contenido analizado se limita a las publicaciones del feed, ${ }^{1}$ dado que su disponibilidad temporal es mayor (y no tan efímera como las de las stories).

Para medir los estereotipos de belleza y aspectos como la posible objetivación sexual e hipersexualización, se ha elaborado una ficha de análisis que incluye todos los indicadores que se pretenden medir vinculados a estereotipos femeninos (hipersexualización/objetivación corporal, apariencia física, pose, ángulo, tipo de publicación, contenido, publicidad y marcas) partiendo de una escala previamente elaborada y utilizada por Tiggemann y Zaccardo (2016) a la que se han añadido la categoría temática y publicidad y marcas.

1 Feed de Instagram: distribución de las publicaciones del usuario, visible según se entra a su perfil, ordenadas de manera temporal, ya sea a modo de cuadrícula todas juntas o de manera individual (adaptación de 40 de Fiebre, s. f. b) 


\section{ESTUDIOS}

\section{DISERTACIONES}

Grupos minoritarios y estigmatizados: diversidad funcional, religiosa, étnica, afectivo-sexual o de identidad de género en la comunicación

ISSN: 1856-9536

Doi: https://doi.org/10.12804/revistas.urosario.edu.co/disertaciones/a.11148

Volumen 15, Número 1 / Enero-junio 2022

Versión PDF para imprimir desde

http://revistas.urosario.edu.co/index.php/disertaciones

Tabla 2. Ficha de análisis

\section{Hipersexualización/objetivación corporal}

Sí

No

En la imagen se enfoca a una parte específica del cuerpo

Se utiliza una pose sexy (ejemplo: guiñando un ojo, arqueando la espalda, mirada provocativa...)

No se ve/apenas se ve la cara o el rostro de la persona

\section{Apariencia física}

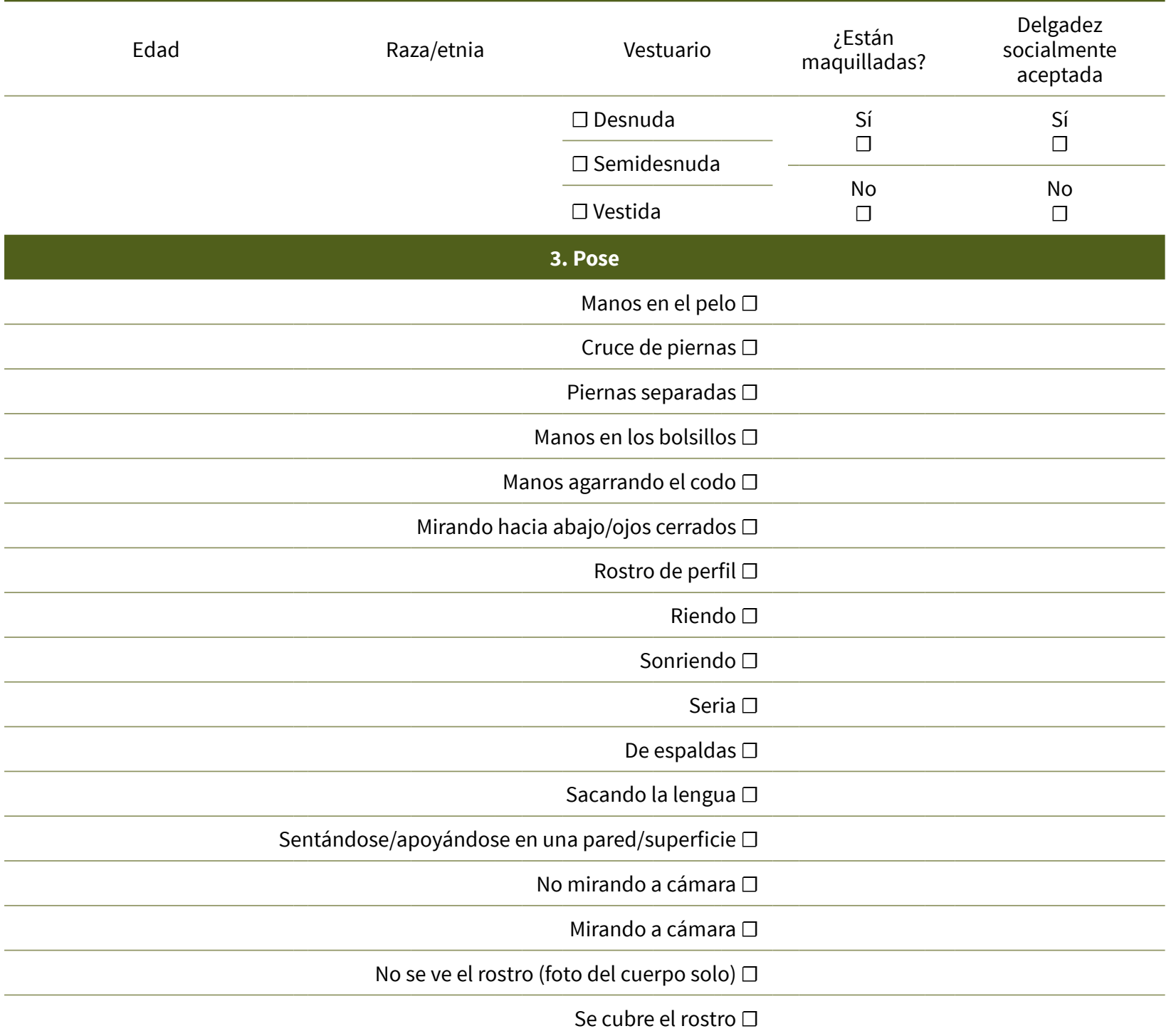




\section{DISERTACIONES}

\section{ESTUDIOS}

Grupos minoritarios y estigmatizados: diversidad funcional, religiosa, étnica, afectivo-sexual o de identidad de género en la comunicación

ISSN: 1856-9536

Doi: https://doi.org/10.12804/revistas.urosario.edu.co/disertaciones/a.11148

Volumen 15, Número 1 / Enero-junio 2022

Versión PDF para imprimir desde

http://revistas.urosario.edu.co/index.php/disertaciones

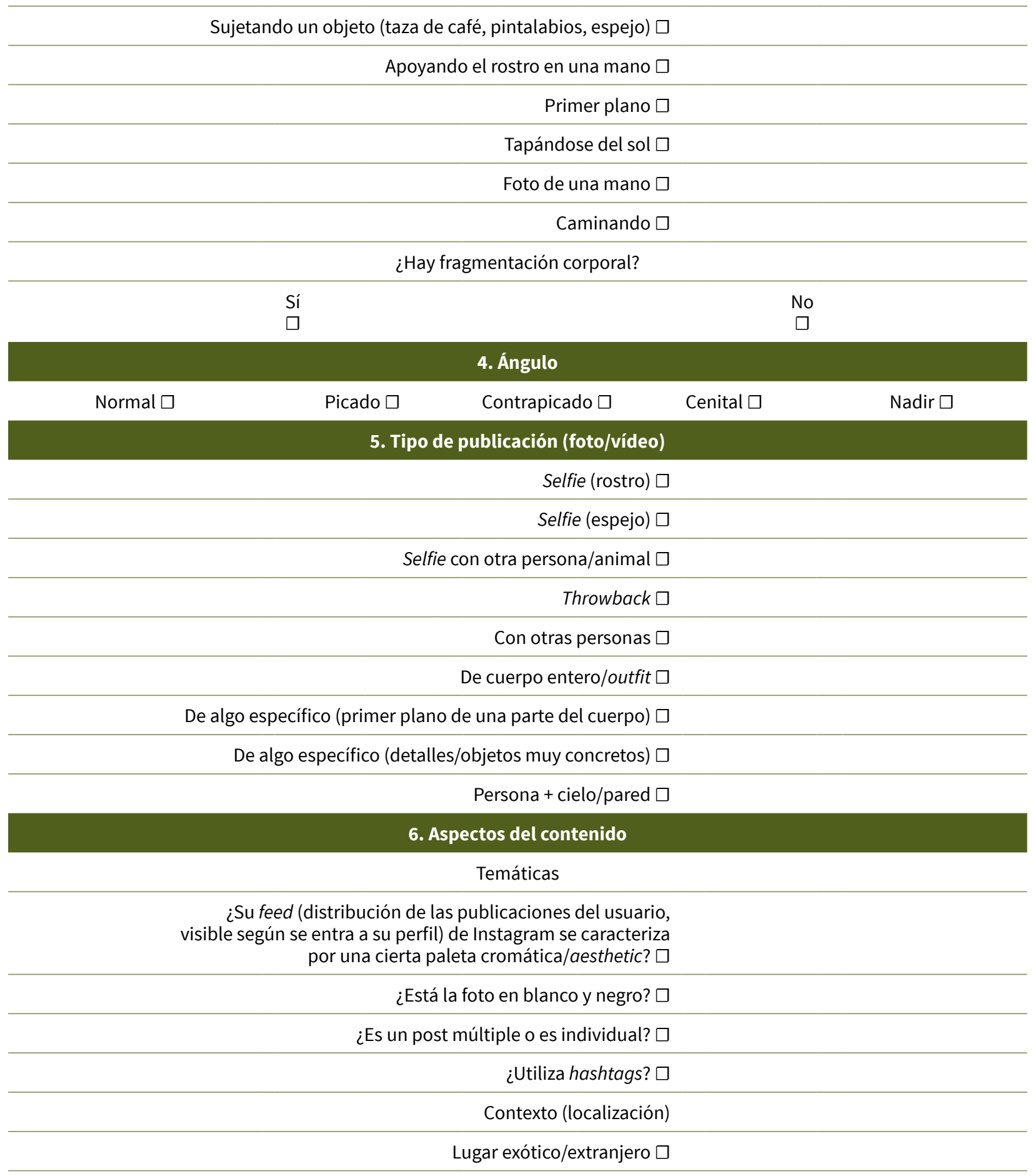

Escenarios cotidianos y accesibles, foto fácil de recrear; lugar de interior (café, casa) $\square$ 


\section{DISERTACIONES}

ESTUDIOS

Grupos minoritarios y estigmatizados: diversidad funcional, religiosa, étnica, afectivo-sexual o de identidad de género en la comunicación

ISSN: $1856-9536$

Doi: https://doi.org/10.12804/revistas.urosario.edu.co/disertaciones/a.11148

Volumen 15, Número 1 / Enero-junio 2022

Versión PDF para imprimir desde

http://revistas.urosario.edu.co/index.php/disertaciones

6. Aspectos del contenido

Pared $\square$

Monumento histórico $\square$

7. Publicidad y marcas

¿Hay presencia publicitaria?

Sí $\square$

No $\square$

¿Qué papel cumple el producto?

Elemento central $\square$

Elemento secundario $\square$

¿Está indicada dicha presencia de publicidad o marcas?

Sí $\square$

No $\square$

Fuente: elaboración propia.

La metodología de este análisis se ha complementado con el desarrollo y distribución online de un cuestionario dirigido a los usuarios de Instagram. El principal objetivo de esta encuesta es entender los motivos por los cuales la gente sigue este tipo de cuentas, observar si existe un determinado grado de consenso sobre los temas reproducidos y analizar qué tipo de publicaciones hace la muestra empleada en Instagram (en su estado de personas anónimas). Es decir, detectar si se reproducen los mismos tipos de contenidos y cánones de belleza que en las cuentas de influencers o de personajes públicos. Este instrumento se creó -y se presentó- en la plataforma Qualtrics, estando disponible desde el 16 de abril del 2021 hasta el día 30 del mismo mes. Dicho cuestionario era anónimo, y ha sido distribuido a una población heterogénea tanto por las redes sociales Twitter e Instagram, como por la aplicación de mensajería instantánea WhatsApp, para poder contar con una mayor participación (sirviéndose de útiles como los retweets o el compartir stories ${ }^{2}$ que inviten a responder a la encuesta). Se adaptó una estrategia de muestreo no probabilístico por bola de nieve (snowball sampling), con el fin de aumentar su alcance y, por tanto, incrementar el tamaño muestral utilizado. Dicho método consiste en pedirle a las personas participantes del estudio que compartieran el link de la encuesta con sus amistades, familiares y contactos (Ochoa, 2015; Baxter \& Babbie, 2004, citados en Igartua et al., 2020).

Antes de colaborar en la investigación, los participantes fueron informados de que el tratamiento de sus datos sería íntegramente anónimo - así como del objetivo del estudio en términos generales-. El cuestionario se estructuró en cinco bloques. El primer bloque contaba con diez ítems o preguntas, el segundo con cuatro, el tercero con cinco y el cuarto con un único ítem. Se establecieron en total 20 preguntas, siendo de naturaleza variada (combinando Likert y dicotómicas), si bien la participación en ciertos bloques estaba condicionada al tipo de respuestas (afirmativas o negativas) que se dieran en algunas preguntas. Estaba compuesto por medidas creadas ad hoc, así como inspirado en preguntas utilizadas en estudios previos (Cáceres et al., 2017; Fernández, 2017). En el bloque 1 ("Influencers") se preguntaba -entre otros- al encuestado si contaba con cuenta en la red social Instagram, si sabía lo que era un influencer o el grado de influencia que tienen sobre él las publicaciones de figuras públicas; en el bloque 2 ("Publicaciones del encuestado"), se trataban - por ejemplo- temas como el tipo de contenidos que

2 Stories: contenidos audiovisuales visibles durante 24 horas desde su publicación, los cuales no permiten ni «me gusta» ni comentarios (adaptación de BBC Mundo, 2016). 


\section{DISERTACIONES}

ESTUDIOS

se publican en redes sociales (publicaciones en el feed, stories o ambas) o la tendencia a compararse con otras personas; en el tercer bloque ("Filtros") se planteaban preguntas sobre el uso de filtros de stories de Instagram que alteran el aspecto físico y en cómo afectan a los usuarios, y en el cuarto ("Percepción de la autoimagen") se indagaba en la autoestima de los encuestados, indicando su grado de acuerdo o desacuerdo con un conjunto de afirmaciones, con base en una escala Likert de 1 a 5 , siendo $1=$ totalmente en desacuerdo y $5=$ totalmente de acuerdo. Con relación a cómo podrían influir las publicaciones de influencers en la persona encuestada (planteada de acuerdo con una escala Likert, en la cual se pidió al usuario que respondiera a una serie de afirmaciones seleccionando un número de 1 a 5 , siendo 1 = en absoluto de acuerdo y 5 = totalmente de acuerdo), se introdujeron las siguientes afirmaciones: "sus decisiones de compra", "imitar estilismos o maquillajes", "posar (copiar sus poses en mis propias publicaciones)" e "impacto negativo en su autoestima y su autoimagen".

El empleo de la encuesta permite medir el impacto de influencia de los influencers, mediante el planteamiento de cuestiones relativas, entre otros, a si suelen copiar los estilismos de estas figuras públicas, si les permiten informarse sobre tendencias en belleza, moda e incluso, si reproducen sus formas de posar o de realizar fotos para poder obtener más "me gusta", así como el estado de su autoimagen y cómo se ve afectado por compartir contenidos en línea con frecuencia.

Contó con una única versión en castellano (difundida íntegramente dentro del territorio español) y, en consecuencia, se accedió a una muestra total - una vez depurada la matriz de datos - de 509 personas, residentes en su totalidad en España. Participaron en la encuesta un total de 80 hombres (15,72\%), 422 mujeres (82,91\%) y 7 personas $(1,38 \%)$ pertenecientes a otro género (del cual se pidió que especificaran: la totalidad de las respuestas dentro de esta categoría declaran ser de género no binario). La media de edad de los encuestados es de 23,61 años $(\sigma=7,71)$.

\section{Resultados}

A continuación, se muestran los principales resultados en relación con la revisión teórica realizada y las hipótesis planteadas sobre estudio de los cánones de belleza en Instagram, partiendo del estudio de las publicaciones compartidas por los usuarios en las redes sociales y de las cuentas de influencers/instagrammers más populares.

\section{$\mathrm{H}_{1}$ : las cuentas de influencers/instagrammers más populares perpetúan el ideal de belleza tradicional (vinculado a la hipersexualización corporal o rasgos caucásicos, entre otros elementos) y pueden influir en la imagen física que proyectan sus seguidores.}

La totalidad de la muestra analizada responde a los principales estereotipos de belleza femenina planteados por Tiggemann y Zaccardo (2016), que han sido tomados como punto de partida del análisis. Las protagonistas de las cuentas analizadas responden a las características de raza caucásica, persona joven que se encuentra dentro de los estándares socialmente aceptables de delgadez. Su media de edad es de 31,4 años $(\sigma=5,60)$ y el $60 \%$ de ellas presentan un feed con una distribución u organización visualmente atractiva. Por otra parte, el tipo de publicación que más se tiende a realizar es el de "cuerpo entero u outfit" (un 48,77\%), seguido de las de "detalles específicos del cuerpo". En el 12,05\% de los casos se efectúa un primer plano de una parte del cuerpo en concreto. La semidesnudez - definida aquí como el hecho de aparecer en ropa interior o de baño, así como con una sola prenda y cubriéndose estratégicamente el resto del cuerpo- se dio en un $17,08 \%$ de los posts; y la desnudez total, 


\section{DISERTACIONES}

ESTUDIOS

sin recurrir a ningún tipo de vestimenta, representaba únicamente un 3,33\% de los materiales estudiados. En cuanto a la presencia de maquillaje en las fotografías, aparece en un $91,67 \%$ de las publicaciones estudiadas. Asimismo, la fragmentación corporal supone un porcentaje de 19,17\% del total del análisis. En este sentido, los datos de la encuesta revelan que el $24,33 \%$ de los encuestados suele subir imágenes tipo "selfies (de mi rostro)", un 17,22\% afirmó subir principalmente "fotos de cuerpo entero" y el 15,33\% reconoció que uno de los tipos de imagen que publicaba eran "selfies (en un espejo)". La encuesta también hizo hincapié en las stories de Instagram y sus filtros. En este caso, un 64,69\% de los encuestados afirmó que les gustaba usar filtros en las selfies de sus historias. Además, un 50,71\% de los encuestados respondió que disfrutaba alterando su físico; concretamente un $40,53 \%$ disfrutaba con los filtros que hacían que la piel pareciera perfecta, un 24,23\% optó por los que aumentan el tamaño de sus ojos o de sus labios, un 14,10\% por cambiar el color de los ojos y el 21,15\% para reducir la nariz.

Atendiendo a estos datos, podría confirmarse que se han hallado ciertos rasgos de hipersexualización y objetivación en las cuentas de influencers analizadas y los datos de la encuesta indican una similitud en cuanto a contenidos de personas influencers y anónimas. Sin embargo, los datos disponibles no son suficientes para la confirmación de la hipótesis.

\section{$\mathrm{H}_{2}$ : el ámbito influencer está ligado al consumo de productos de moda y belleza.}

Los datos analizados muestran que las temáticas que más predominan en este tipo de cuentas son la moda y belleza, ya sea mediante la promoción de bienes o servicios de esta índole, o a través de la subida de contenidos a Instagram en los que se centre el foco de atención en sus estilismos. Los productos de moda o belleza promocionados ocupan mayoritariamente una posición o papel central $(71,25 \%)$ dentro de los propios contenidos.
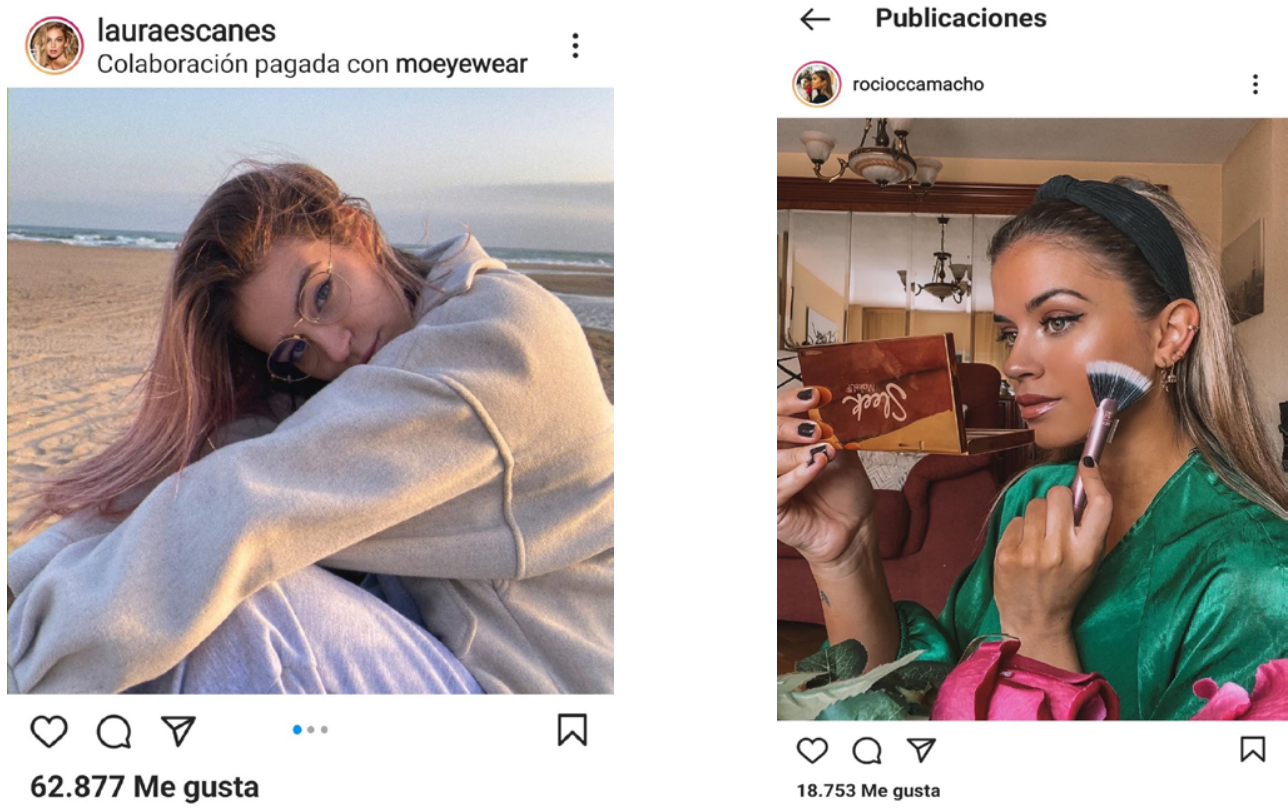

Figura 1. Publicaciones de Laura Escanes y Rocío Camacho

Fuente: @lauraescanes, @rocioccamacho. 


\section{DISERTACIONES}

ESTUDIOS

Al completar estos resultados con los datos obtenidos mediante la encuesta, se aprecia que los contenidos publicados en este tipo de cuentas influyen en ámbitos como el poder que tienen en las adquisiciones -y consecuentes decisiones de compra- de las personas encuestadas. Estos parámetros cuentan con una significancia de 2,49 sobre 5 en lo relativo a su peso en dichas decisiones $(\sigma=1,10)$. Con respecto a la posible influencia de las redes sociales en los hábitos de consumo de los encuestados, se preguntó si habían adquirido algo (tanto en los últimos doce meses como en un intervalo temporal general, representado por "alguna vez", ya sea dicha adquisición una prenda de vestir, un artículo de belleza o un objeto de cualquier otra índole), únicamente por haberlo visto previamente a un influencer en redes sociales. En ese marco, un 51,21\% respondió de manera afirmativa - dentro de la pregunta enfocada en el marco de tiempo de los últimos doce meses-, mientras que, si trasladamos esto a un periodo cronológico indefinido, el porcentaje de gente que responde que sí es del 69,36\%.

Tras analizar estos datos, la segunda hipótesis queda confirmada al poder comprobarse que el consumo de productos de belleza y moda suponen el $71 \%$ del contenido publicado por algunas de las influencers más destacadas.

$\mathrm{H}_{3}$ : los influencers de este ámbito crean un tipo que refuerza los estereotipos femeninos y que influye en los patrones de conducta de usuarios anónimos de esta red social.

Tras analizar la pose de las influencers, otro de los parámetros establecidos por Tiggemann y Zaccardo (2016) para medir la presencia de estereotipos, es el ángulo de la cámara. En este caso, los resultados obtenidos revelan que las influencers aparecen "mirando a cámara" en un $16,65 \%$ de los posts. No lo hacen en un $11,24 \%$ y tienden a mostrarse "apoyadas o sentadas en paredes u otras superficies" en un 10,16\% de las fotos o vídeos presentes en esta investigación. También es necesario recalcar que sus rostros presentan una ligera tendencia a estar "sonriendo" (8,54\%), "riendo" (5,30\%) o "serios" (8,22\%), y a menudo se fotografían sujetando objetos (como pintalabios, tazas de café, entre otros). Esta categoría abarca un 7,89\% de la muestra. En cuanto a los ángulos, los resultados denotan una cierta predisposición a recurrir a los de tipo "normal" (56,67\%), mientras que solo se usan los calificados como "picado" y "contrapicado" en un $26,25 \%$ y un $16,67 \%$-respectivamente- del conjunto muestral con el que se ha trabajado.

Así, los datos obtenidos en la encuesta parecen indicar que los contenidos desarrollados en Instagram ejercen cierto impacto en las personas que siguen estas cuentas. Para conocer cómo podrían influir las publicaciones de influencers en las personas encuestadas (con base en una escala Likert, en la cual se pidió al usuario que respondiera a una serie de afirmaciones seleccionando un número de 1 a 5 , siendo 1 "en absoluto de acuerdo" y 5 "totalmente de acuerdo"), se introdujeron las siguientes afirmaciones: "sus decisiones de compra", "imitar estilismos o maquillajes", "posar (copiar sus poses en mis propias publicaciones)" e "impacto negativo en su autoestima y su autoimagen". 


\section{ESTUDIOS}

Grupos minoritarios y estigmatizados: diversidad funcional, religiosa, étnica, afectivo-sexual o de identidad de género en la comunicación

ISSN: $1856-9536$

Doi: https://doi.org/10.12804/revistas.urosario.edu.co/disertaciones/a.11148

Volumen 15, Número 1 / Enero-junio 2022

Versión PDF para imprimir desde

http://revistas.urosario.edu.co/index.php/disertaciones
Sus decisiones de compra

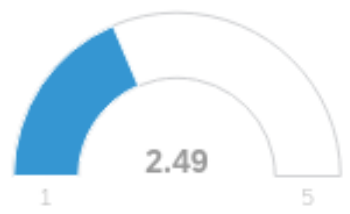

data
Imitar estilismos o maquillajes

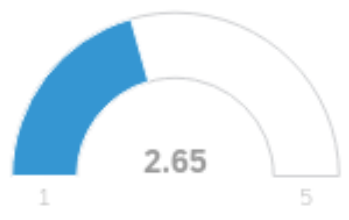

data
Posar (copiar sus poses en mis propias publicaciones)

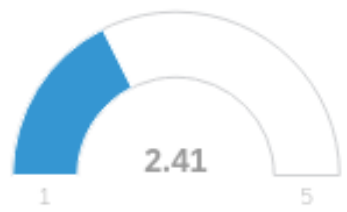

data
Impacto negativo en su autoestima y

autoimagen

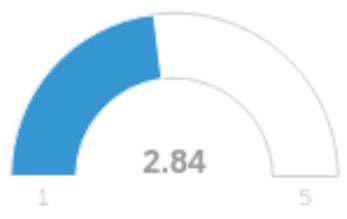

data

Figura 2. Influencia de las publicaciones de influencers en los usuarios

Fuente: elaboración propia.

Dicho esto, se concluyó que las mayores medias (calculadas sobre el número 5) se hallaban en las opciones "impacto negativo en su autoestima y su autoimagen" $(2,84, \sigma=1,46)$, "imitar estilismos o maquillajes" (2,65, $\sigma=1,27)$ y "sus decisiones de compra" $(2,49, \sigma=1,10)$.

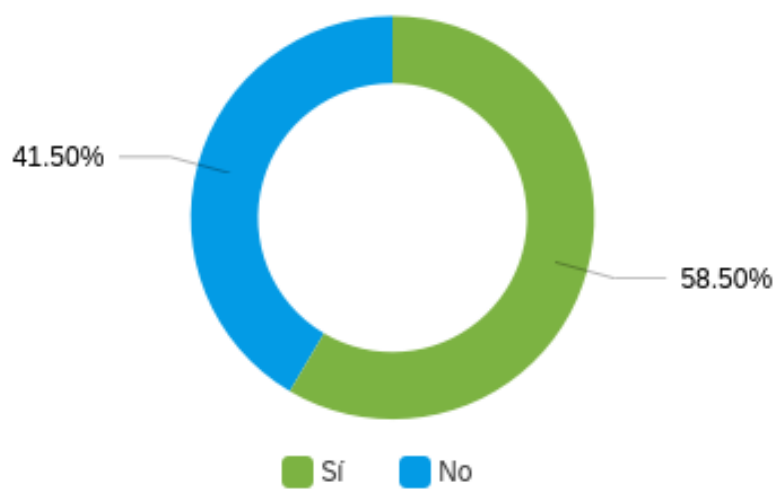

Figura 3. "¿Tiende a compararse con lo que ve en Instagram, ya sea publicado por personajes públicos o por personas anónimas?"

Fuente: elaboración propia a partir de las respuestas obtenidas tras el uso de la plataforma Qualtrics. 


\section{ESTUDIOS}

\section{DISERTACIONES}

Grupos minoritarios y estigmatizados: diversidad funcional, religiosa, étnica, afectivo-sexual o de identidad de género en la comunicación

ISSN: $1856-9536$

Doi: https://doi.org/10.12804/revistas.urosario.edu.co/disertaciones/a.11148

Volumen 15, Número 1 / Enero-junio 2022

Versión PDF para imprimir desde

http://revistas.urosario.edu.co/index.php/disertaciones

La encuesta también inquirió en la predisposición a compararse con los demás, ya sean tanto personajes públicos como personas anónimas, a raíz de lo que se haya visto en línea. El 58,50\% de la muestra afirmó hacerlo. Ante la pregunta de cómo les afectaba el hecho de llevar a cabo dichas comparaciones, un $44,16 \%$ escogió la opción de "impacta negativamente a mi autoimagen, siento que mi atractivo físico es menor que el suyo", y que un $27,16 \%$ ve cómo impacta "negativamente" a su autoestima. Solo un $13,45 \%$ reconoció que impactaba "positivamente a su autoimagen" y que se sentían "más motivados" a cambiar su físico o a quererse más. Por su parte, un $11,17 \%$ opinó que impactaba "positivamente" a su autoestima, viéndose más motivados a "seguir sus sueños" y "hacer lo que más les guste".

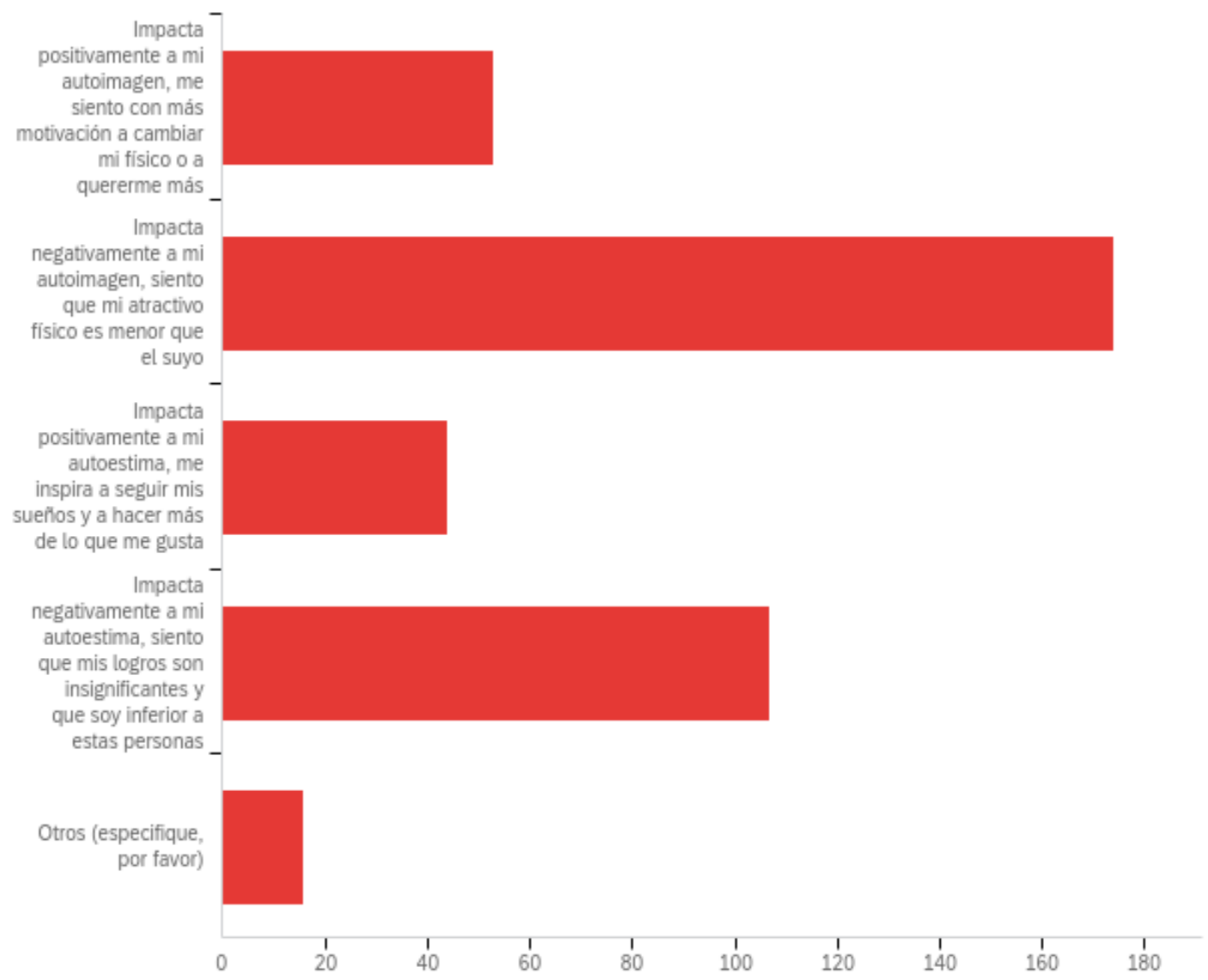

Nota. Distribución de frecuencias de los participantes

Figura 5. Impacto provocado por la comparación con los contenidos de influencers

Fuente: elaboración propia a partir de las respuestas obtenidas tras el uso de la plataforma Qualtrics.

Tras analizar estos resultados, puede afirmarse que la hipótesis quedaría corroborada. 


\section{DISERTACIONES}

ESTUDIOS

Grupos minoritarios y estigmatizados: diversidad funcional, religiosa, étnica, afectivo-sexual o de identidad de

género en la comunicación

ISSN: $1856-9536$

Doi: https://doi.org/10.12804/revistas.urosario.edu.co/disertaciones/a.11148

Volumen 15, Número 1 / Enero-junio 2022

Versión PDF para imprimir desde

http://revistas.urosario.edu.co/index.php/disertaciones

\section{Conclusiones}

El consumo de redes sociales no ha parado de crecer en los últimos años, especialmente entre las personas más jóvenes para quienes, además de un espacio de entretenimiento, se han convertido en la principal fuente de información (Linares, 2021). Pero más allá de la posible sobreexposición a estos nuevos canales digitales, su uso (a menudo) se vincula con un posible impacto en la imagen corporal de la audiencia. Estos son solo algunos motivos por los que se hace más necesario que nunca analizar - desde diversas ópticas- la evolución de las redes sociales, el tipo de contenido que se publica en ellas y a sus protagonistas.

Por eso, el punto de partida de este estudio fue el análisis de algunas de las cuentas de influencers/instagrammers más populares a fin de determinar si podían perpetuar el ideal de belleza tradicional. Los datos analizados procedentes del análisis de contenido y de la encuesta fueron coincidentes con el ideal de belleza femenino tradicional y reflejaron la preocupación por la perfección física, tanto de las propias influencers como de sus seguidores, mediante el uso de maquillaje, filtros y retoques.

Por otra parte, la hipersexualización y la objetivación corporal resultan aspectos fundamentales a la hora de analizar cánones y estereotipos femeninos. Los datos obtenidos en el análisis de contenido reflejaron una cierta presencia de cuerpos semidesnudos (17,8\%). En el caso de la encuesta, el 24,33\% de los encuestados reflejaron su preferencia por publicar fotos con fragmentación corporal, centradas en el rostro en este caso, tipo selfie. En este sentido, aunque no ha podido comprobarse el predominio de la hipersexualización o de fragmentación corporal, los datos indican una tendencia hacia la perpetuación de determinados estereotipos femeninos a través del físico, como la preocupación por el aspecto físico y la manera de mostrarse al resto.

Las temáticas tratadas en las publicaciones de las influencers analizadas y las preferencias de temas de las personas encuestadas también son elementos esenciales para comprobar qué tipo de tópicos se tratan en estos perfiles sociales y qué posibles relaciones pueden darse con la perpetuación del estereotipo femenino que, a menudo, se asocia con aspectos como la preocupación por la moda y la belleza. En este caso, la muestra utilizada para iniciar el análisis "El Estudio de los 500 Españoles más Influyentes" ya refleja que dentro de los cincuenta influencers más importantes de nuestro país hay tan solo ocho hombres. Todas las cuentas analizadas pertenecían a mujeres. En el caso de la encuesta, además, la mayoría de las personas participantes $(82,91 \%)$ fueron mujeres.

Por su parte, los productos de moda y belleza constituyen el elemento central de las publicaciones seleccionadas para el análisis de contenido. En este caso, los datos obtenidos en la encuesta indican que estos contenidos sí influyen en las decisiones de compra de los seguidores (un 69,36\% afirmaba haber hecho compras de productos después de haberlos visto en redes sociales).

Más allá de la creación y perpetuación de estereotipos, el uso de las redes sociales puede ejercer una importante influencia en los patrones de conducta de usuarios anónimos, impactando incluso en aspectos como la autoestima. Tras desarrollar este estudio, ha podido comprobarse que los usuarios anónimos tienden a establecer comparaciones con los influencers que siguen en las redes sociales. A este respecto, los encuestados no solo manifestaron su interés en imitar contenidos y poses vistas en las publicaciones de influencers; también reconocieron que tendían a compararse con el contenido visto en esas cuentas (un 58,5\% de la muestra reconoció hacerlo). Por su parte, el 44,16\% reflejaron que los contenidos vistos en los perfiles de Instagram de estas cuentas impactan de manera negativa a su autoimagen, sintiendo que su atractivo físico "es menor que el suyo".

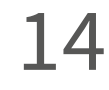




\section{DISERTACIONES}

ESTUDIOS

Grupos minoritarios y estigmatizados: diversidad funcional, religiosa, étnica, afectivo-sexual o de identidad de

género en la comunicación

ISSN: $1856-9536$

Doi: https://doi.org/10.12804/revistas.urosario.edu.co/disertaciones/a.11148

Volumen 15, Número 1 / Enero-junio 2022

Versión PDF para imprimir desde

http://revistas.urosario.edu.co/index.php/disertaciones

A estos datos es importante añadir la relevancia que adquiere la validación externa -traducida en la obtención de muchos comentarios y likes-, la cual resulta en sentimientos positivos y una mayor confianza. En cuanto a la posible influencia de los comentarios y "me gusta" en los posts de los encuestados, en cómo se sentirían, las afirmaciones "con más confianza y más feliz" y "mi autoestima aumenta" obtuvieron, respectivamente, una puntuación de 3,65 sobre $5(\sigma=1,17)$ y de 3,59 $(\sigma=1,17)$. Estos datos enfatizan la importancia de tener que realizar un uso sano y saludable de las redes sociales, consistente principalmente en evitar las comparaciones.

En cuanto a las limitaciones de investigación encontradas a lo largo de su desarrollo, debemos hacer mención de que los datos obtenidos no permiten establecer una relación causa-efecto lo suficientemente sólida (por ejemplo, en el caso del análisis de contenido, se observa que, si bien se da hipersexualización y objetivación en las cuentas de influencers analizadas, el conjunto de datos trabajado no nos permite confirmar la primera hipótesis). Es decir, hubiera sido conveniente una metodología de mayor complejidad, para poder contar con algo más que un reporte descriptivo de los usos de Instagram por parte de los participantes. Sin embargo, hay que resaltar su utilidad a la hora de obtener una perspectiva y un primer acercamiento al impacto que pueden tener fenómenos como los influencers en la creación o mantenimiento de estereotipos.

Así mismo, este trabajo abre la puerta a futuras investigaciones en las que puedan explorarse otros aspectos de las redes sociales desde la óptica del género; también puede extrapolarse a la representación de ciertos colectivos o minorías.

\section{Referencias}

1. 40 deFiebre (s. f.). ¿Qué es el feed de Instagram? Diccionario de Marketing 40deFiebre. 40 de Fiebre. https:// www.40defiebre.com/que-es/feed-instagram

2. Baltar, F., Gorjup, M. T., \& Pàmies, M. (2011). Aplicación de muestreo virtual y diseños mixtos en el estudio de poblaciones ocultas. Actas de la Conferencia Iberoamericana de Investigación Cualitativa, 92-106. http://nulan.mdp.edu.ar/1429/

3. ввс Mundo. (2016). Qué es "Historias" de Instagram y en qué se diferencia de Snapchat. BBc. https://www. bbc.com/mundo/noticias-36962958

4. Cáceres, G., Perea da Silva, T., Vázquez, M., \& Torres, M. G. (2017). El impacto de los influencers de Instagram en la decisión de compra de indumentaria de los jóvenes. [Trabajo Final de Investigación, Universidad Argentina de la Empresa]. Repositorio Institucional UADE. https://repositorio.uade.edu.ar/xmlui/ handle/123456789/6331

5. Daldorph, B. (2017). New French law says airbrushed or photoshopped images must be labelled. France 24. https://www.france24.com/en/20170930-france-fashion-photoshop-law-models-skinny

6. Feltman, C. E., \& Szymanski, D. M. (2018). Instagram use and self-objectification: The roles of internalization, comparison, appearance commentary, and feminism. Sex Roles, 78(5), 311-324. https://doi.org/10.1007/ s11199-017-0796-1

7. Fernández Lerma, A. (2017). Estudio del origen de la figura del influencer y análisis de su poder de influencia en base a sus comunidades [Tesis de pregrado, Universitat Pompeu Fabra]. Repositorio upF. http://hdl. handle.net/10230/36313 


\section{DISERTACIONES}

ESTUDIOS

Grupos minoritarios y estigmatizados: diversidad funcional, religiosa, étnica, afectivo-sexual o de identidad de

género en la comunicación

ISSN: $1856-9536$

Doi: https://doi.org/10.12804/revistas.urosario.edu.co/disertaciones/a.11148

Volumen 15, Número 1 / Enero-junio 2022

Versión PDF para imprimir desde

http://revistas.urosario.edu.co/index.php/disertaciones

8. Fernández Pardo, A. (2013). El backstage de los egoblogs. Ediciones Protocolo.

9. Festinger, L. (1954). A theory of social comparison processes. Human Relations, 7(2), 117-140. https://doi. org/10.1177/001872675400700202

10. Fredrickson, B. L., \& Roberts, T. (1997). Objectification theory: Toward understanding women's lived experiences and mental health risks. Psychology of Women Quarterly, 21(2), 173-206. https://doi. org/10.1111/j.1471-6402.1997.tb00108.x

11. Fundación Marqués de Oliva. (2021). Estudio sobre las 500 personas más influyentes de España. Fundación Marqués de Oliva. https://cutt.ly/qjRRvlv

12. García, S. (2021, 9 de febrero). Adiós al engaño de la piel perfecta: El Reino Unido prohíbe los filtros de belleza a los influencers en Instagram. S Moda El país. https://smoda.elpais.com/belleza/ filtros-belleza-influencers-instagram/

13. Garlick, H. (2020). Why gender stereotypes are perpetuated on Instagram. FT Magazine. https://www.ft.com/ content/2cc5ca3a-6337-11ea-a6cd-df28cc3c6a68

14. Igartua, J. J., Ortega-Mohedano, F., \& Arcila-Calderón, C. (2020). Usos de la comunicación en los tiempos del Coronavirus. Un estudio transcultural. El Profesional de la Información, 29(3), e290318. https://doi. org/10.3145/EPI

15. Jiang, S., \& Ngien, A. (2020). The effects of Instagram use, social comparison, and self-esteem on social anxiety: A survey study in Singapore. Social Media + Society, 6(2). https://doi.org/10.1177/2 056305120912488

16. Linares, H. (2021, 6 de mayo). Solo uno de cada tres jóvenes de entre 16 y 24 años sabe identificar las fuentes de lo que lee en redes sociales. Kantar. https://www.kantar.com/es/inspiracion/publicidad-y-medios/ solo-uno-de-cada-tres-jovenes-de-entre-16-y-24-anos-sabe-identificar-la-fuente

17. Keyte, R., Mullis, L., Egan, H., Hussain, M., Cook, A., \& Mantzios, M. (2020). Self-compassion and Instagram use is explained by the relation to anxiety, depression, and stress. Journal of Technology in Behavioral Science, 6(2), 436-441. https://doi.org/10.1007/s41347-020-00186-z

18. Lim, M., \& Yang, Y. (2015). Effects of users' envy and shame on social comparison that occurs on social network services. Computers in Human Behavior, 51, 300-311. https://doi.org/10.1016/j.chb.2015.05.013

19. Lowe-Calverley, E., Grieve, R., \& Padgett, C. (2019). A risky investment? Examining the outcomes of emotional investment in Instagram. Telematics and Informatics, 45, 101299. https://doi.org/10.1016/j. tele.2019.101299

20. Lup, K., Trub, L., \& Rosenthal, L. (2015). Instagram \#instasad?: Exploring associations among Instagram use, depressive symptoms, negative social comparison, and strangers followed. Cyberpsychology, Behavior, and Social Networking, 18(5), 247-252. https://doi.org/10.1089/cyber.2014.0560

21. Martín García, T. (2017). Los blogs de moda: nuevos preservadores de los estereotipos de género de las revistas femeninas [Tesis de Doctorado, Universidad Complutense de Madrid]. Repositorio Institucional de la ucm. https://eprints.ucm.es/id/eprint/42086/

22. McLean, S. A., Paxton, S. J., Wertheim, E. H., \& Masters, J. (2015). Photoshopping the selfie: Self photo editing and photo investment are associated with body dissatisfaction in adolescent girls. The International Journal of Eating Disorders, 48(8), 1132-1140. https://doi.org/10.1002/eat.22449 


\section{DISERTACIONES}

ESTUDIOS

Grupos minoritarios y estigmatizados: diversidad funcional, religiosa, étnica, afectivo-sexual o de identidad de

23. Menéndez Menéndez, I., \& Figueras Maz, M. (2014). La Evolución de la prensa femenina en España: de "La Pensadora Gaditana” a los blogs. Comunicación: Revista de Recerca i D’anàlisi, 30(1), 25-48. https://dialnet. unirioja.es/servlet/articulo?codigo $=4364729$

24. O'Brien, K. (2015). The cultivation of eating disorders through Instagram. University of South Florida. https://scholarcommons.usf.edu/etd/6004

25. Ortiz, M. I. (2021). Reino Unido prohíbe los filtros de belleza en Instagram a las influencers. ABc. https://www. abc.es/estilo/belleza/abci-reino-unido-prohibe-filtros-belleza-instagram-influencers-202102101905_ noticia.html

26. Porta, L., \& Silva, M. (2003). La investigación cualitativa: el análisis de contenido en la investigación educativa. Anuario Digital de Investigación Educativa, (14), 28. http://biblioteca.esucomex.cl/RCA/La\%20 investigaci\%C3\%B3n\%20cualitativa_el\%20an\%C3\%A1lisis\%20de\%20contenido\%20en\%20la\%20investigaci\%C3\%B3n\%20educativa.pdf

27. Reece, A. G., \& Danforth, C. M. (2017). Instagram photos reveal predictive markers of depression. EPJ Data Science, 6, 1-12. https://doi.org/10.1140/epjds/s13688-017-0110-z

28. Riera, S., \& Figueras Maz, M. (2012). El modelo de belleza de la mujer en los blogs de moda. ¿Una alternativa a la prensa femenina tradicional? Medios de Comunicación, Publicidad y Género, 7. http://revpubli. unileon.es/ojs/index.php/cuestionesdegenero/article/view/908

29. Rodríguez, M. P., Pando-Canteli, M. J., \& Zeberio, M. B. (2017). ¿Generan estereotipos de género los medios de comunicación?: Reflexión crítica para educadores. DEusto Social Impact Briefings, 1. https://doi. org/10.18543/dsib-1(2016)-pp89-115.pdf

30. Ruiz Molina, E. (2014). Los blogs de moda; del periodismo al marketing. Análisis de las interacciones entre los egoblogs, los blogs periodísticos y las industrias del sector [Tesis de Doctorado, Universitat Autònoma de Barcelona]. https://www.tesisenred.net/handle/10803/362641

31. Smith, R. H., \& Kim, S. H. (2007). Comprehending envy [Entendiendo la envidia]. Psychological Bulletin, 133(1), 46. https://doi.org/10.1037/0033-2909.133.1.46

32. Tiggemann, M., \& Zaccardo, M. (2016). "Strong is the new skinny": A content analysis of \#fitspiration images on Instagram. Journal of Health Psychology, 23(8), 1003-1011. https://doi.org/10.1177/1359105316639436

33. Tiggemann, M., \& Barbato, I. (2018). “You look great!”: The effect of viewing appearance-related Instagram comments on women's body. Body Image, 27, 61-66. https://doi.org/10.1016/j.bodyim.2018.08.009

34. Tiggemann, M., Hayden, S., Brown, Z., \& Veldhuis, J. (2018). The effect of Instagram “likes” on women's social comparison and body dissatisfaction. Body Image, 26, 90-97. https://doi.org/10.1016/j.bodyim.2018.07.002

35. Tolentino, J. (2019). The Age of Instagram Face: How social media, FaceTune, and plastic surgery created a single, cyborgian look. The New Yorker. https://www.newyorker.com/culture/decade-in-review/ the-age-of-instagram-face 